\title{
A PTP4A3 Peptide PIMAP39 Modulates TNF-Alpha Levels and Endotoxic Shock
}

\author{
Xiaoren Tang Timothy Woodward Salomon Amar \\ Department of Periodontology and Oral Biology, Boston University Goldman School of Dental Medicine, \\ Boston, Mass., USA
}

\section{Key Words}

Immunology $\cdot$ In vitro assays $\cdot$ Inflammation $\cdot$ Kinases . Regulation of lethal toxin - MAP kinases - Phosphatases . Signal transduction $\cdot$ LITAF $\cdot$ Phosphorylation $\cdot$ Peptide $\cdot$ PIMAP39 • TNF

\begin{abstract}
Lipopolysaccharide (LPS) stimulation of macrophages initiates intracellular signaling pathways leading to activation of MAPK and its subsequent influence on cytokine production. We recently identified a LITAF-STAT6(B) complex regulated by $\mathrm{p} 38$ MAPK in response to LPS stimulation. However, the LPS-induced cascade in the p38/LITAF/TNF signaling pathway remains unclear. Here, we identified PTP4A3, a protein tyrosine phosphotase, as a novel negative regulator of LPSinduced LITAF/TNF- $\alpha$ production. PTP4A3 exerts its negative role by dephosphorylating p38 $\alpha$ MAPK in response to LPS stimulation of primary macrophages. PTP4A3 expression is upregulated in primary macrophages. Further structure-function analysis revealed that a unique short peptide (PIMAP39) derived from PTP4A3 is capable of mimicking the functionality of full-length PTP4A3 to selectively dephosphorylate p38 $\alpha$ and indirectly suppress LPS-induced LITAFSTAT6B complex when it is translocated from the cytoplasmic region to the nucleus of the cell. Treatment of mice with
\end{abstract}

PIMAP39 significantly attenuates the severity of adverse host responses to LPS stimulation, and in some cases provides complete resistance to a lethal dose of LPS due to suppression of TNF- $\alpha$ production. All together, these results reveal a previously unrecognized role for the PTP4A3 pathway in response to LPS.

Copyright $\odot 2009$ S. Karger AG, Basel

\section{Introduction}

Lipopolysaccharide (LPS) is a major integral structural component of the outer membrane of Gram-negative bacteria and activates monocytes and macrophages to produce cytokines such as TNF- $\alpha$ [1]. TNF- $\alpha$, a primary cytokine mediator of immune regulation and inflammation, has been implicated as a multifaceted factor with both beneficial and detrimental functions, generating both pro-inflammatory and apoptotic effects in various cell types [2].

LPS stimulates intracellular signaling pathways by regulating activation of cytoplasmic signaling proteins including tyrosine kinases [3-6]. Recently, we found that the LITAF-STAT6B complex plays a major role in transcription of several inflammatory cytokines including TNF- $\alpha[7,8]$, and activation of p38 MAPK is required for

\section{KARGER}

(C) 2009 S. Karger AG, Basel

Fax +4161306 1234

E-Mail karger@karger.ch

www.karger.com www.karger.com/jin
Prof. Salomon Amar

Department of Periodontology and Oral Biology

Boston University Goldman School of Dental Medicine

650 Albany Street, X-343, Boston, MA 02118 (USA)

Tel. +1 617638 4820, Fax +1 617638 4974, E-Mail samar@bu.edu 
LITAF gene expression in response to LPS stimulation [9]. Several studies have indicated that p38 MAPK is activated in differentiating myocytes, where NF- $\mathrm{BB}$ activity is present [10], but suppressed by protein tyrosine phosphatases (PTPs). In particular, dual-specificity protein phosphatases are known to play a key role in regulation of p38 MAPK activity [11-14]. Altogether, these findings underscore the role of PTPs to significantly modify the biological outcome of LPS-induced pro-inflammatory response in macrophages.

Previous work from our laboratory using the OneHybrid System (Clontech) identified PTP4A3 (also called phosphatase of regenerating liver3, PRL, 1, 2 and 3) [1517] as a potential binding protein to LITAF promoter (data not shown). Based on our interest in modulating LITAF transcriptional activity, we have pursued this avenue and investigated its involvement in LPS-induced LITAF/TNF- $\alpha$ production. Our study indicates that PTP4A3/LITAF protein-DNA interaction inhibits LITAF promoter activity. In addition, PTP4A3 regulates dephosphorylation of $\mathrm{p} 38 \alpha$ MAPK in THP-1 or primary macrophage cells in response to LPS stimulation. Furthermore, a specific short peptide of PTP4A3, PIMAP39, is found to mimic the function of the full-length PTP4A3 for its ability to selectively dephosphorylate p38 $\alpha$ and to indirectly downregulate LITAF/TNF- $\alpha$ production in response to LPS stimulation. This unique peptide sequence derived from PTP4A3 is highly conserved among species and unexpectedly is cell permeable both in an in vitro cell culture and in vivo systems. Treatment of animals with PIMAP39 peptide decreases the severity of the adverse host responses to LPS stimulation, and in some cases provides complete resistance to an otherwise lethal dose of LPS. All together, these results reveal a novel and previously unrecognized role for PTP4A3 in LPS signaling.

\section{Experimental Procedures}

\section{Cell Culture}

All bacterial cloning constructs used Escherichia coli strain DH5 $\alpha$ (Invitrogen). U2OS human osteosarcoma cells (wild type) were grown in DMEM supplemented with $10 \%$ fetal bovine serum (FBS). THP-1 cells (TIB-202; American Type Culture Collection) or mouse primary macrophages were grown in RPMI 1640 supplemented with $10 \%$ FBS. All cell cultures were maintained in a $37^{\circ} \mathrm{C}$ humidified atmosphere containing $5 \% \mathrm{CO}_{2}$. E. coli LPS was purchased from Sigma-Aldrich. Both ultra pure E. coli LPS (a TLR4 ligand, catalog No. tlrl-pelps) and Pam3CSK4 (a TLR2 ligand, catalog No. tlrl-pms) were purchased from InvivoGen.
Primary Macrophages

Primary macrophages were obtained from C57BL/6 (wildtype) mice (The Jackson Laboratory) and purified by conventional methods [18].

Mice

All mice (wild type) were 8-12 weeks of age and were maintained under strict pathogen-free conditions at the Boston University transgenic facility. All procedures involving animals were approved by the Institutional Animal Care and Use Committee at Boston University Medical Center.

\section{Kinase Inhibitors}

AG490, U0126 and SB203580 were purchased from EMD Biosciences. Sodium orthovanadate was purchased from Sigma-Aldrich. Human THP-1 cells were treated with $10 \mu \mathrm{M}$ sodium orthovanadate (a global PTP inhibitor used for PTP4A3) [19], $20 \mu \mathrm{M}$ AG490 (JAK inhibitor) [20], $20 \mu \mathrm{M}$ U0126 (ERK inhibitor) [21] and $20 \mu \mathrm{M}$ SB203580 (p38 MAPK inhibitor) [22].

\section{PCR or RT-PCR}

PCR or RT-PCR was performed using the QIAQuick RNA miniprep kit (Qiagen) and Platinum ${ }^{\circledR}$ Quantitative RT-PCR ThermoScript ${ }^{\mathrm{TM}}$ One-Step System kit (Invitrogen) following the manufacturers' instructions.

\section{Plasmid Constructs}

The full-length LITAF promoter DNA, pGLP990, was used as described [23]. The PTP4A3 DNA clone (catalog No. MHS101159590) provided by Open Biosystems was used as template and amplified by PCR with the primer pairs $5^{\prime}$-atggctcggatgaaccgcccg-3' and 5'-ctacataacgcagcaccgggt- $3^{\prime}$. The in-frame DNA fragment of PTP4A3 (BC003105, amino acid (a.a.) 1-149) and a series of PTP4A3 deletion DNAs (del) from No. 1 to No. 9 (No. 1: a.a.102-149, No. 2: 55-149, No. 3: 39-149, No. 4: 1-120, No. 5: 1-93, No. 6: 1-66, No. 7: 1-38, No. 8: 54-86, No. 9: 39-66) was then subcloned into the pcDNA3HA vector [8], but 1 methionine was added for initiation, as shown in figure 2 . The NF- $\kappa \mathrm{B}$ promoter DNA (NT_082868) was amplified by PCR with mouse normal tissue genomic DNA (BioChain) as template plus the primer pairs $5^{\prime}$-atggacggtgaggctgccetct-3' and 5'-tgaaagggaaagacaaggcac- $3^{\prime}$, then inserted into pGL3-basic vector (Promega) and named pGL3NFkBP.

DNA Transfection in Cells

LPS-treated or untreated THP-1 cells or mouse primary macrophage cells were transfected with DNAs $\left(1 \mu \mathrm{g} / 1 \times 10^{5}\right.$ cells/ well) or untreated as controls by Lipofectamine reagent (Invitrogen) over night.

RNA Interference of PTP4A3

THP- 1 cells $\left(5 \times 10^{6}\right)$ were untreated or treated with $0.1 \mu \mathrm{g} / \mathrm{ml}$ LPS (Sigma) for $3 \mathrm{~h}$ and washed with PBS. Cells were then transfected by Oligofectamine (Invitrogen) with $100 \mathrm{nM}$ of small interfering RNA (siRNA) specific for PTP4A3 (named PTP4A3RNAi, with duplex sequences: GUACGAGGACGCCAUCCAGUU and AACUGGAUGGCGUCCUCGUAC, designed and synthesized by Invitrogen) and $100 \mathrm{nM}$ of nonspecific siRNA (named NSRNAi) [8] as control following the manufacturer's instructions. The treated and untreated cells were grown in RPMI 1640 medi- 
um supplemented with $10 \% \mathrm{FBS}$ and maintained at $37^{\circ} \mathrm{C}$ in $5 \%$ $\mathrm{CO}_{2}$ overnight. The protein lysate from the treated and untreated cells were analyzed by Western blotting.

Peptides

Synthetic peptides were supplied by Biosynthesis Inc. PIMAP39 consisted of the PTP4A3 sequence KYGATTVVRVCEVTYDKTPLEKDGITVV located in the region from amino acids 39 to 66 . SCpep served as a negative control peptide and consisted of the randomly scrambled sequence VTGKLTDTEVVTAYVIDEPKYVCRVTGK (Medusa Random Sample Generator Software, Randombots.com). Both peptides were solubilized in DMSO immediately prior to use.

\section{Fluorescence Microscopy}

Fluorescein 5-isothiocyanate (FITC)-labeled PIMAP39 was supplied by Biosynthesis Inc. FITC-PIMAP39 was solubilized in DMSO and delivered into mouse primary macrophages or mouse circulatory system. For macrophage samples, cells $\left(5 \times 10^{3}\right)$ from 3 -month-old mice were seeded over cover slips $(22 \mathrm{~mm}$; VWR Scientific) in 6 -well plates at $37^{\circ} \mathrm{C}$ and $5 \% \mathrm{CO}_{2}$ overnight. After washing with PBS, the cells were treated with $500 \mathrm{ng} / \mathrm{ml}$ FITCPIMAP39 (B) or DMSO (A) as control. Cells were continuously incubated in RPMI 1640 supplemented with $10 \% \mathrm{FBS}$ at $37^{\circ} \mathrm{C}$ and $5 \% \mathrm{CO}_{2}$ overnight. The cover slips were removed from the wells and the attached cells were stained with $50 \mathrm{~nm}$ LysoTracker Red DND-99 (Invitrogen) for $1.5 \mathrm{~h}$ and then air-dried. For tissue section samples, mice were treated with $1 \mathrm{mg}$ of FITC-PIMAP39 (E and F) or DMSO (C and D) as control by tail vein injection as described [24]. One hour after injection, mice were sacrificed and their livers were immediately harvested. Harvested livers were set in uniform orientations in molds using Histoprep (Fisher). Tenmicrometer-thick cross-sections were cut and transferred with a paintbrush to glass slides followed by hematoxylin and eosin staining. Cross-sections were made at $-24^{\circ} \mathrm{C}$ using $\mathrm{HM} 505 \mathrm{E}$ cryostat (Microm). Both the treated cells and sections above were exposed to visible light and fluorescent light by Olympus BX40 microscope at magnifications of $200 \times(\mathrm{A}$ and $\mathrm{B})$ or $1,000 \times(\mathrm{C}-$ F). Images were taken with a MicroFIRE camera under uniform exposure time ( $1 \mathrm{~s}$ for fluorescent light, $30 \mathrm{~ms}$ for visible light). The analysis of these images was performed using the software Image-Pro plus 5.0 (MediaCybernetics).

\section{Immunoprecipitation}

The lysate from the treated cells were incubated with $2 \mu \mathrm{g}$ of the appropriate antibody for $2 \mathrm{~h}$ at $4^{\circ} \mathrm{C}$, followed by incubation with $20 \mu \mathrm{l}$ of protein A/G PLUS-Agarose-Sepharose beads (SC2003; Santa Cruz Biotechnology) for an additional hour. The beads were washed 3 times in PBS buffer and then suspended in SDS sample buffer heated at $95^{\circ} \mathrm{C}$ for $5 \mathrm{~min}$. The eluted proteins were applied to SDS-polyacrylamide gels.

\section{Western Blot Analysis}

Western blot was done following the manufacturers' instructions with antibodies: LITAF (611615; BD Biosciences), Actin (C11; Santa Cruz Biotechnology), HA (sc-805), p38 (sc-535), p-p38 (sc-7973), NF-kBp65 (sc-7151), p-NF-kBp65 (sc-33020-R) and p-Akt1/2/3 (sc-7985-R). Protein fractionation (whole cell lysate, cytosol and nucleus fractions) was done using the ProteoExtract Subcellular Proteome Extraction Kit according to the manufac- turer's instructions (Calbiochem). The proteins were then detected by Western blotting with the antibody against FITC (sc-65218; Santa Cruz Biotechnology) or $\beta$-tubulin ( $\beta$-tubulin was used as a diagnostic marker due to its expression only in nucleus, sc-5274; Santa Cruz Biotechnology).

\section{ELISA}

ELISA was done following the manufacturer's instructions (Abraxis). ELISA immunoreactivity was quantified by using a microplate reader (Bio-Rad) and graphed. Triplicate assays were performed followed by statistical analysis.

\section{Endotoxic Shock Assay}

At the age of 8-12 weeks, weight-matched wild-type mice $(\mathrm{n}=$ 15) weighing 20-25 g were injected intraperitoneally with lethal dose of LPS (12.5 ng LPS + $1 \mathrm{mg}$ D-Gal per gram body weight), followed immediately by tail vein injection of peptides or DMSO as control. The treated mice were maintained in a normal light cycle room and provided with free access to rodent chow and water, and were monitored for their behavior and mortality every hour. The survival time of each treated mouse was recorded and a Kaplan-Meier graph was made based on the results. For blood plasma, blood was collected every $2 \mathrm{~h}$ after injection for a total duration of $6 \mathrm{~h}$. To avoid causing death by blood loss, the duration of blood collection did not extend past $6 \mathrm{~h}$. Mice were warmed under a heating lamp to promote blood flow and a small incision was made on the tail. About 10-50 $\mu$ l of blood was collected per animal at each time point $(2,4$ or $6 \mathrm{~h})$. The blood samples at each time point from mice within the same groups were pooled in order to have enough blood for ELISA. Red blood cells were removed from the sample via centrifugation at 5,000 rpm for $1 \mathrm{~min}$ using serum separator tubes (Fisher). Pooled plasma samples from each group were measured by ELISA (Abraxis) according to the manufacturer's instructions. ELISA immunoreactivity was quantified by using a microplate reader (Bio-Rad) and the results from the 6-hour mark were graphed.

Statistical Analysis

Statistical analysis was performed using Student's t test. The differences were considered statistically significant at $\mathrm{p} \leq 0.05$.

\section{Results}

\section{Effects of Overexpression of PTP4A3 or Its Derived}

Deletions on LPS-Induced TNF- $\alpha$ Production

As shown in figure 1a, transient transfection of PTP4A3 caused a 77.2\% decrease in LITAF promoter-activated luciferase gene expression compared with the controls. To investigate a link between PTP4A3 and the LPS signaling cascade, THP-1 cells were cotreated with $0.1 \mu \mathrm{g} /$ $\mathrm{ml}$ E. coli LPS and/or various inhibitors, sodium orthovanadate (a global PTP inhibitor used for PTP4A3), AG490 (JAK inhibitor), U0126 (ERK inhibitor), SB203580 (p38 MAPK inhibitor) or untreated as control. The supernatants from cell culture at each time point $(2,4,6,8,10,12$, 


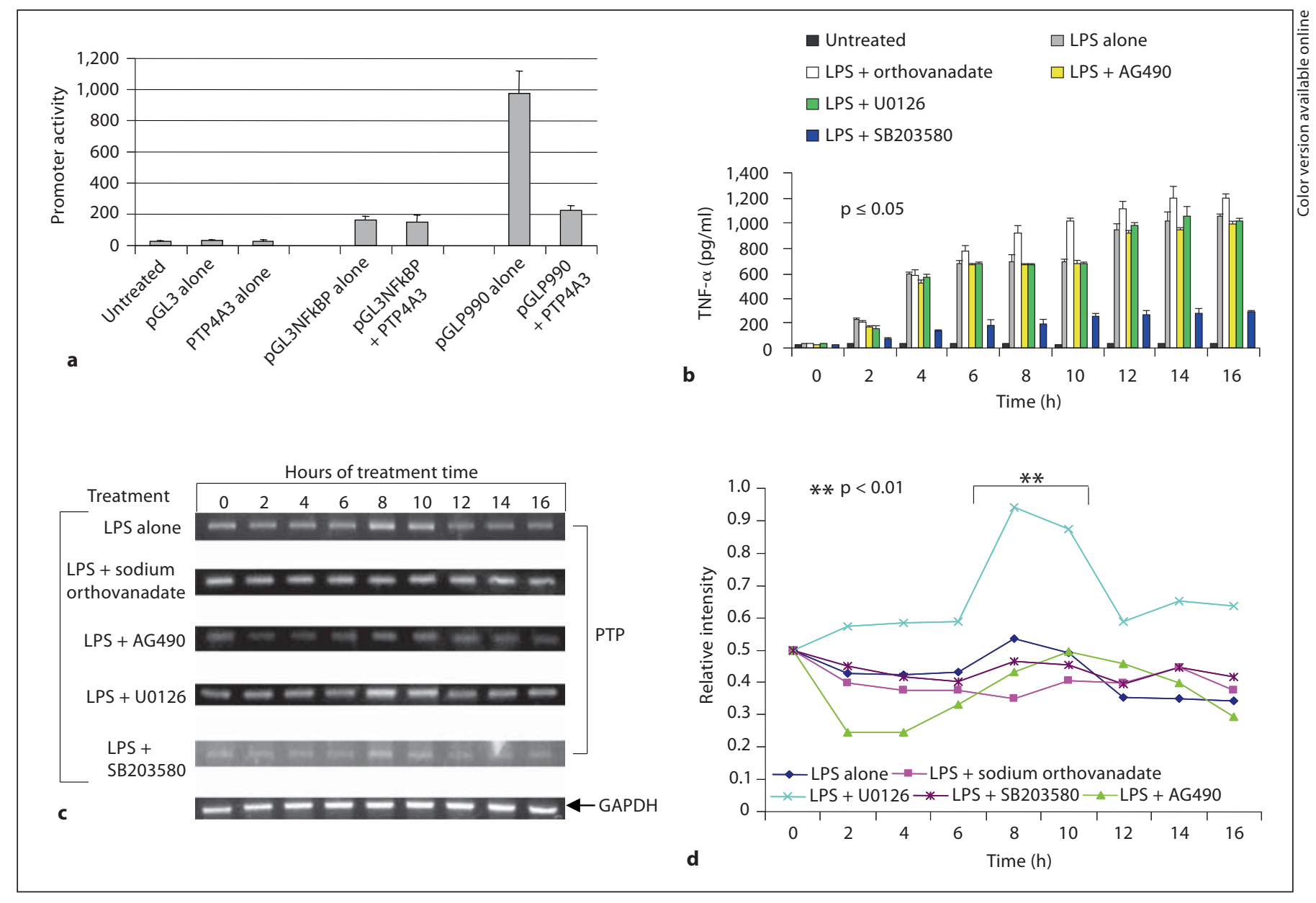

Fig. 1. PTP4A3-induced downregulation of LITAF promoter activity and effects of inhibitors on LPS-induced PTP4A3 production. U2OS cells were transiently transfected with DNAs including pGLP990, or pGL3-basic and pGL3NFkBP and untreated as controls using Lipofectamine 2000 (Invitrogen). The protein extracts $(30 \mu \mathrm{g})$ from each culture of treated cells were analyzed by luciferase assay. The full-length LITAF promoter (pGLP990) yielded the highest luciferase activity, which was set at $100 \%$ for comparison of segments. pGLP990 plus PTP4A3 significantly reduced LITAF promoter activity to $22.8 \%$ (a). THP-1 cells were differentiated with $100 \mathrm{nM}$ phorbol-12 myristate- 13 acetate and seeded $\left(1 \times 10^{5}\right)$ in 6 -well plates overnight. After washing with PBS, cells were stimulated with $0.1 \mu \mathrm{g} / \mathrm{ml}$ E.coli LPS for $3 \mathrm{~h}$. The cells were washed with PBS again and treated with various inhibitors, 10 M sodium orthovanadate, $20 \mu \mathrm{M}$ AG490, $20 \mu \mathrm{M}$ U0126, $20 \mu \mathrm{M}$ SB203580 or untreated as control. $200 \mu \mathrm{l}$ of supernatant was collected for ELISA assay (b) from each treatment at 2, 4, 6, 8, $10,12,14$ and $16 \mathrm{~h}$ and its corresponding mRNA was prepared for RT-PCR (c) by first extracting total RNA from the treated cells using the QIAQuick RNA miniprep kit (Qiagen). One microgram of each RNA was used to generate cDNA with Platinum Quantitative RT-PCR ThermoScript One-Step System kit (Invitrogen), 10 ng of cDNA from each test was analyzed by PCR using PTP4A3 forward primer 5'-ATGGCTCGGATGAACCGC-3' and reverse primer $5^{\prime}$ CTACATAACGCAGCACCG-3'. Another nanogram of cDNA was analyzed by PCR using GAPDH primer pairs (Invitrogen) as control. Densitometric analysis (d) of RT-PCR (c) with intensity values normalized to GAPDH and using the zero hour value as reference intensity by using VersaDoc (Bio-Rad). These intensity values were further statistically analyzed by Student's t test utilizing Microsoft Excel which showed that PTP4A3 expression at 8$10 \mathrm{~h}$ after treatment with LPS was significant.
14 and $16 \mathrm{~h}$ after stimulation) were collected and the potential role of these inhibitors on TNF- $\alpha$ production was measured by ELISA (Abraxis). In parallel, mRNA from each experimental group was prepared and quantified by RT-PCR. As shown by ELISA (fig. 1b), with the exception of treatment with the inhibitor SB203580, which substantially reduced LPS-induced TNF- $\alpha$ expression, all other inhibitor treatments, AG490, U0126 or sodium orthovanadate, did not affect LPS-induced TNF- $\alpha$ expression compared to LPS alone. Interestingly, within 8-10 h after 
Fig. 2. Diagram of PTP4A3 DNA constructs. Different lengths of PTP4A3 DNA were truncated and inserted into the pcDNA3HA vector [8]. Full-length wildtype PTP4A3 (white box), named pcHA PTP, and its derived segments (gray box, No. 1-9) are shown. The synthetic peptide representing amino acids 39-66, named PIMAP39, and its randomly scrambled peptide as a negative control, named SCpep, are shown.

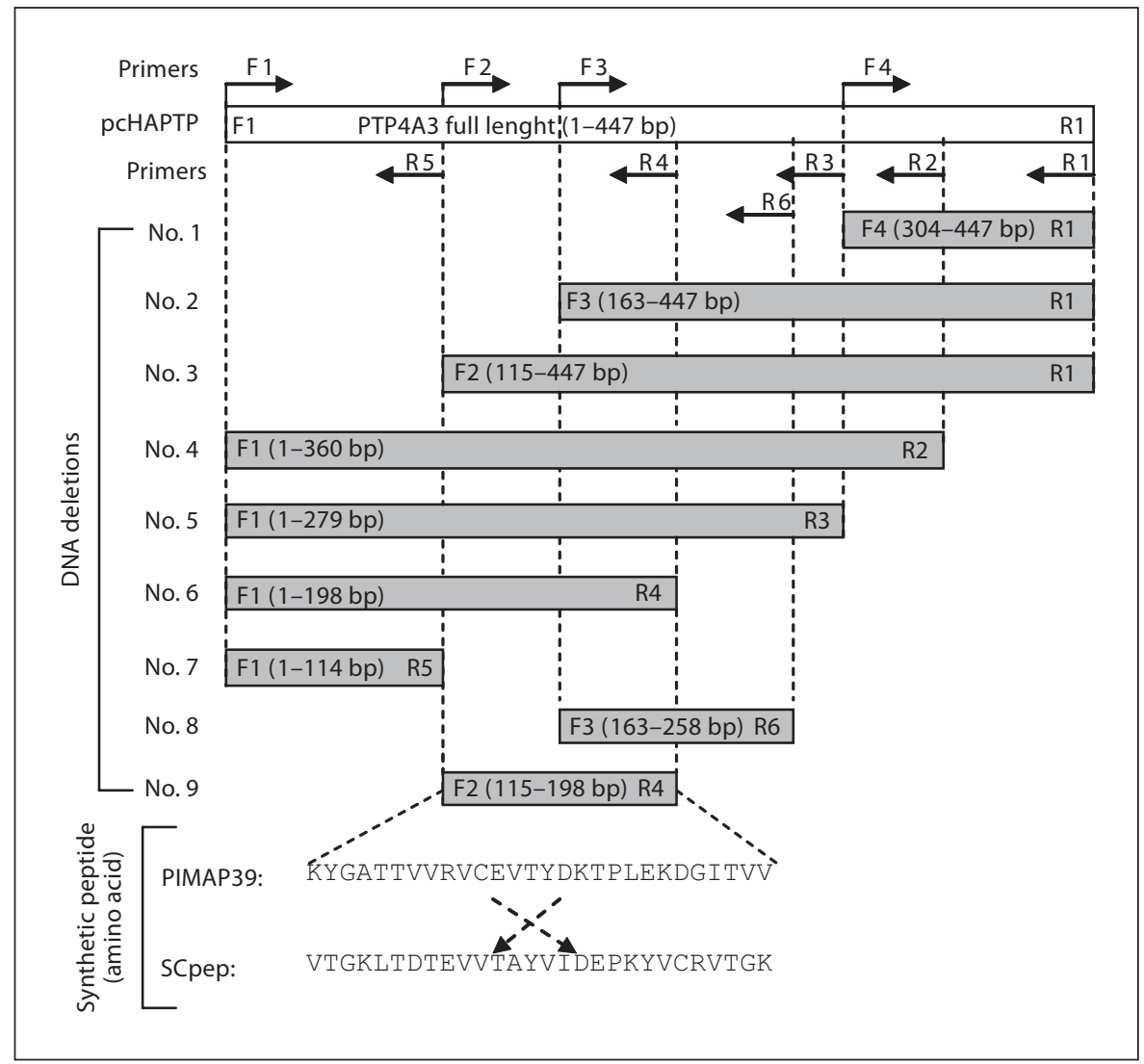

treatment, TNF- $\alpha$ levels in response to LPS alone or LPS + sodium orthovanadate-treated cells increased much more than in LPS + AG490- or LPS + U0126-treated cells. Additionally, the concentration of secreted TNF- $\alpha$ by the LPS + sodium orthovanadate-treated cells was on average $20 \%$ higher than in cells treated only with LPS at the 8hour mark and beyond (fig. 1b). Curiously, RT-PCR analysis showed that PTP4A3 expression was at a maximum between 8-10 h after treatment with LPS alone or plus inhibitor, AG490, U0126 or SB203580. No such maximum occurred with the LPS + sodium orthovanadate-treated cells (fig. $1 c$ and $d$ ). This suggests that PTP4A3 is a LPSinduced short-lived protein regulating TNF- $\alpha$ levels, and its expression is not affected by JAK, ERK or p38 MAPK, because inhibition of these kinases did not alter PTP4A3 gene expression in response to LPS. To investigate the possible involvement of PTP4A3 in LPS-induced TNF- $\alpha$ production in THP-1 cells, we treated these cells with E. coli LPS and/or DNA constructs (fig. 2). The ELISA analysis showed that overexpression of these constructs significantly downregulates LPS-induced TNF- $\alpha$ production (fig. 3a), provided they contained the a.a.39-66 residue, such as with pcHAPTP (a.a.1-149), No. 3 (a.a.39149), No. 4 (a.a.1-120), No. 5 (a.a.1-93), No. 6 (a.a.1-66) and No. 9 (a.a.39-66). Lacking this sequence (No. 1, a.a.102-149; No. 7, a.a.1-38) or containing only a partial residue (No. 2, a.a.55-149; No. 8, a.a.55-86) has failed to sufficiently downregulate TNF- $\alpha$ production compared to controls. The same result was obtained in mouse primary macrophage cells [unpubl. data]. These data suggest that the ability of PTP4A3 to regulate the LPS-induced TNF- $\alpha$ production requires the presence of this specific residue from a.a.39-66.

\section{Effects of PTP4A3 and Its Derived Deletions on p38 $\alpha$ /LITAF Signaling Pathway}

We recently found that LITAF remains inactivated in the absence of p38 MAP kinase (p38 $\alpha$ ) and that the inhibition of p38 $\alpha$ downregulates cytokines such as TNF- $\alpha$ [9]. Based on the findings described above, we hypothesized that PTP4A3 functions within this LPSdependent $\mathrm{p} 38 \alpha /$ LITAF signaling pathway. To test this hypothesis, THP-1 cells (fig. 3b) were cotreated with $E$. coli LPS plus pcDNA3 DNA as control (fig. 3b, lane 2) 
and PTP4A3 DNA (fig. 3b, lane 3). The lysate protein from each treated cell culture was analyzed by Western blot using our anti-HA antibody detecting an overexpression of PTP4A3 (lane 3), an anti-p-p38 antibody showing a significant reduction of $\mathrm{p} 38 \alpha$ phosphorylation (lane 3) and an anti-p38 antibody showing unaffected p $38 \alpha$ protein levels at the same time (lane 3). Also, a reduction of LITAF was observed under the aforementioned conditions (lane 3, LITAF detection). Additionally, the PTP4A3 overexpression had no effect on both the protein and phosphorylation levels of NF- $\kappa \mathrm{B}(\mathrm{p} 65)$ and Akt1/2/3 (lane 3) compared to the controls (lanes 1 and 2). This suggests that PTP4A3 mediates dephosphorylation of $\mathrm{p} 38 \alpha$ and consequently leads to the inhibition of LPS-induced LITAF production. Similar results in mouse primary macrophage cells are presented (fig. 3c). To further characterize the role of endogenous PTP4A3 on p38 $\alpha$ phosphorylation, we knocked down PTP4A3 expression using RNAi. Silencing PTP4A3 expression significantly increased $\mathrm{p} 38 \alpha$ phosphorylation in response to LPS (fig. 3d, lane 3). Consistent with the role of PTP4A3 in p38 $\alpha$ phosphorylation, overexpression of PTP4A3 reduced LPS-induced phosphorylation of p38 $\alpha$ (fig. $4 \mathrm{~d}$, lane 4 ), while blocking PTP4A3 expression with RNAi suppressed its ability to dephosphorylate p38 (fig. $4 \mathrm{~d}$, lane 5), suggesting that in vivo, PTP4A3 is an endogenous regulator of $\mathrm{p} 38$ in response to LPS stimulation. To further corroborate the role of PTP4A3 in modulation of phosphorylation of $\mathrm{p} 38 \alpha$, we tested whether PTP4A3 physically interacts with $\mathrm{p} 38 \alpha$. To this end, mouse primary macrophages were transiently transfected with PTP4A3, and the binding ability of PTP4A3 to endogenous $\mathrm{p} 38 \alpha$ was determined by immunoprecipitation. The result showed that PTP4A3 indeed binds to p38 $\alpha$, further underscoring a critical role for PTP4A3 in negative regulation of $\mathrm{p} 38 \alpha$ activity (fig. $3 e$, lane 4 ) as described [25].

\section{Effects of PIMAP39 on p38 $\alpha /$ LITAF Signaling}

Pathway

A short peptide (named PIMAP39; fig. 2) was synthesized with the amino acid sequence from a.a.39-66 (KYGATTVVRVCEVTYDKTPLEKDGITVV) of PTP4A3, the region which we found to function as an inhibitor of TNF- $\alpha$ production, as described above (fig. 3a). An FITCtagged PIMAP39, was also synthesized in order to detect and establish that the peptide could be delivered into cells or the circulatory system. As shown in figure 4, FITC-labeled PIMAP39, delivered into primary macrophages or delivered by injection into mice, was clearly present in both the treated primary macrophages (fig. $4 \mathrm{~b}$ ) and the white blood cells within the liver sections (fig. $4 \mathrm{~g}$ ) when observed with fluorescent light compared to the cells treated with DMSO alone (fig. 4a and e). This suggests that the FITC-labeled PIMAP39 peptide enters the cells and tissues via circulating blood to produce the fluorescent signal both in vitro and in vivo. Additionally, PIMAP39 was also found to be present in the cytoplasmic region of primary macrophages after $5 \mathrm{~h}$ of peptide treatment as described (fig. 4c). To further examine the effects of PIMAP39 on LPS-induced TNF- $\alpha$ production, a time course analysis was performed. THP-1 cells (wild type) and the primary macrophage cells (LITAF-/-) were treated with E. coli LPS plus PIMAP39 or SCpep as control. The supernatants from each cell culture at designated time points $(2,5$ and $8 \mathrm{~h}$ after stimulation) were collected and measured by ELISA (fig. 5a) and the corresponding lysate protein was detected by Western blot with antibodies against p-p38 $\alpha, \mathrm{p} 38$ or LITAF, or actin as control (fig. 5b). It is clear that PIMAP39-treated cells secreted significantly lower amounts of TNF- $\alpha$ (fig. 5a), coinciding with a markedly lower p $38 \alpha$ phosphorylation level and lower amount of LITAF protein level (fig. 5b, lane 5) compared to the controls (fig. 5b, lane 2 or 6). Importantly, LITAF-deficient macrophages after treatment with PIMAP did not demonstrate a reduction in LPS-induced TNF- $\alpha$ production compared to untreated or SCpeptreated controls (fig. 5a). Furthermore, in wild-type peritoneal mouse macrophages, the addition of PIMAP39 did not change activation/phosphorylation of JNK, ERK, p65 or IRF3 in response to either TLR4 ligand or TLR2 ligand, while PIMAP39 significantly affected LITAF activation (fig. 5c).

\section{The Effect of PIMAP39 on in vivo LPS-Induced}

Endotoxic Shock

To investigate how PIMAP39 mediates endotoxic shock, 3-month-old weight-matched mice were treated with a lethal dose of LPS (12.5 ng LPS + $1 \mathrm{mg}$ D-Gal per gram body weight), followed immediately by a tail vein injection of PIMAP39 or controls. The treated mice were monitored for their behavior and mortality every hour. The survival time of each treated mouse was measured and graphed (fig. 6a). Most control mice (LPS + DMSO and LPS + SCpep) became sick at 3-4 h and deaths occurred between 6-8 h (fig. 6a, black and green lines). Treatment with LPS plus $40 \mu \mathrm{g} / \mathrm{g}$ PIMAP39 (fig. 6a, red line) delayed sickness and prolonged survival time by an average of $4 \mathrm{~h}$ compared to the controls (fig. 6a, black and green lines). Surprisingly, despite being administered a 

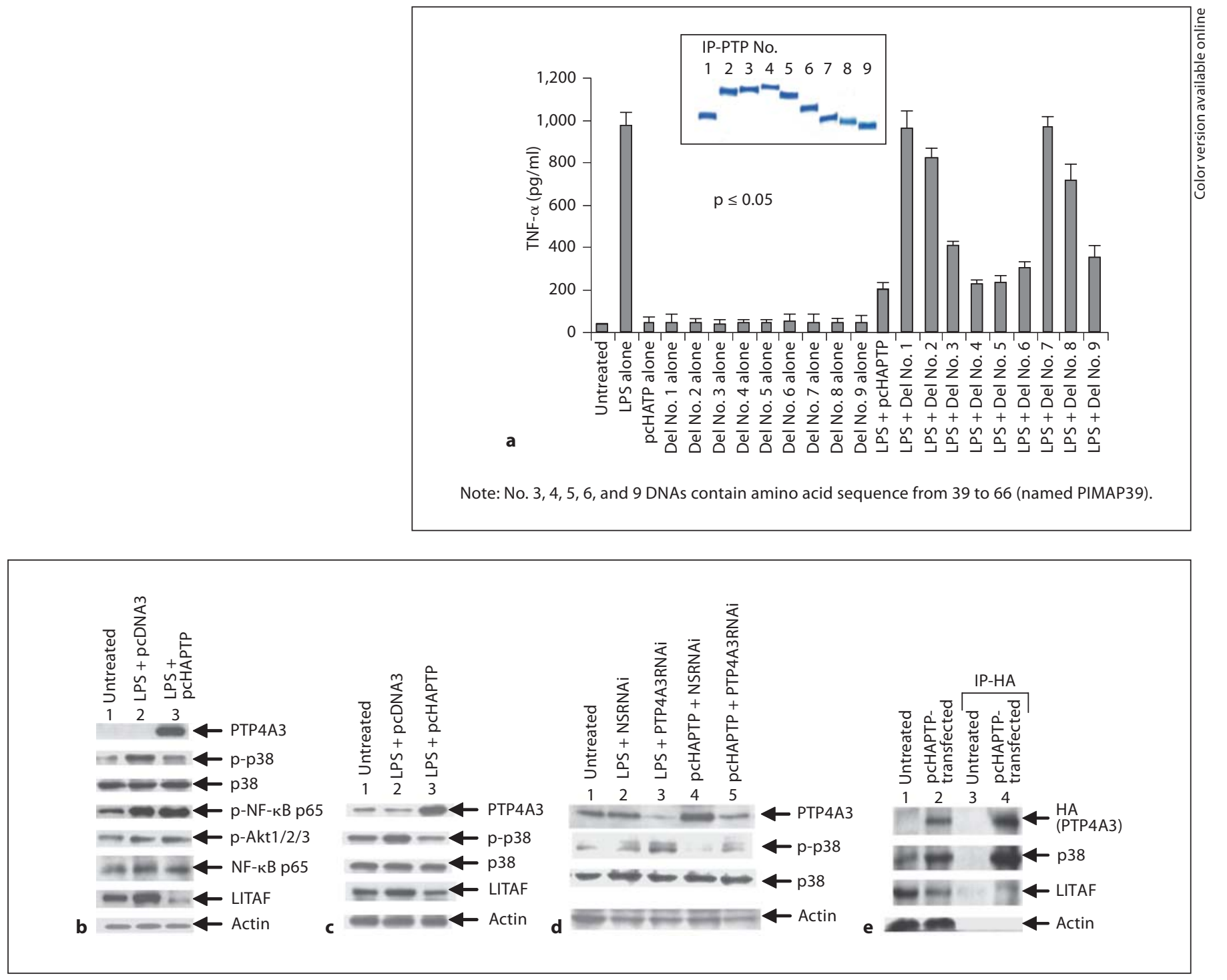

Fig. 3. Effects of PTP DNAs on LPS-induced TNF- $\alpha$. THP-1 cells $\left(1 \times 10^{5}\right)$ were stimulated with $0.1 \mu \mathrm{g} / \mathrm{ml} \mathrm{E}$. coli LPS for $3 \mathrm{~h}$. The cells were washed and transiently transfected with DNAs as above using Lipofectamine reagent (Invitrogen) over night. The supernatants from each culture were measured by ELISA (Abraxis). ELISA immunoreactivity was quantified (a). To show equal amounts of expression of the different PTP4A3 constructs (fig. 2), their extracts were purified by immunoprecipitation (IP) with HA. One microgram of IP proteins was run, stained by Coomassie Blue and attached (a). THP-1 cells (b) or mouse primary macrophage cells (c) were stimulated with $0.1 \mu \mathrm{g} / \mathrm{ml} \mathrm{E}$. coli LPS alone for $3 \mathrm{~h}$ and then transfected with $1 \mu \mathrm{g} / \mathrm{ml} \mathrm{pcHAPTP}$ DNA (lane 3) or with $1 \mu \mathrm{g} / \mathrm{ml} \mathrm{pcDNA}$ (lane 2) and untreated (lane 1) as controls by Lipofectamine reagent over night and lysed. The lysate proteins were used for Western blot with antibodies against LITAF (611614; BD Biosciences) and PTP4A3 (named PRL-3, sc-21581; Santa Cruz Biotechnology), p38 (sc-535), p-p38 (sc-7973), p65 (sc-7151), p-p65 (sc-33020-R), p-Akt1/2/3 (sc-7985-R) and Actin (sc-1615). For further analysis by RNAi of PTP4A3 (d), THP-1 cells were stimulated (lanes 2 and 3) or untreated (lanes 1, 4 and 5) with $0.1 \mu \mathrm{g} / \mathrm{ml}$ LPS (Sigma), then transfected by 0.5. $\mu \mathrm{g}$ pcHAPTP (lanes 4 and 5) plus $100 \mathrm{nM}$ PTP4A3RNAi (lanes 3 and 5) or $100 \mathrm{nM}$ NSRNAi as control (lanes 2 and 4), and incubated over night. The protein lysate from treated and untreated cells were used for Western blot with antibodies against PRL-3, p38, p-p38 and actin. Mouse primary macrophages (e) were transiently transfected with no DNA (lane 1), or $1 \mu \mathrm{g}$ of DNA of HA-tagged pcHAPTP (lane 2) using Lipofectamine reagent, and their extracts were purified by IP with HA. One microgram of IP proteins (lanes 3 and 4) and unpurified proteins as control (lanes 1 and 2) were detected with antibodies against HA, LITAF, p38 and actin. 

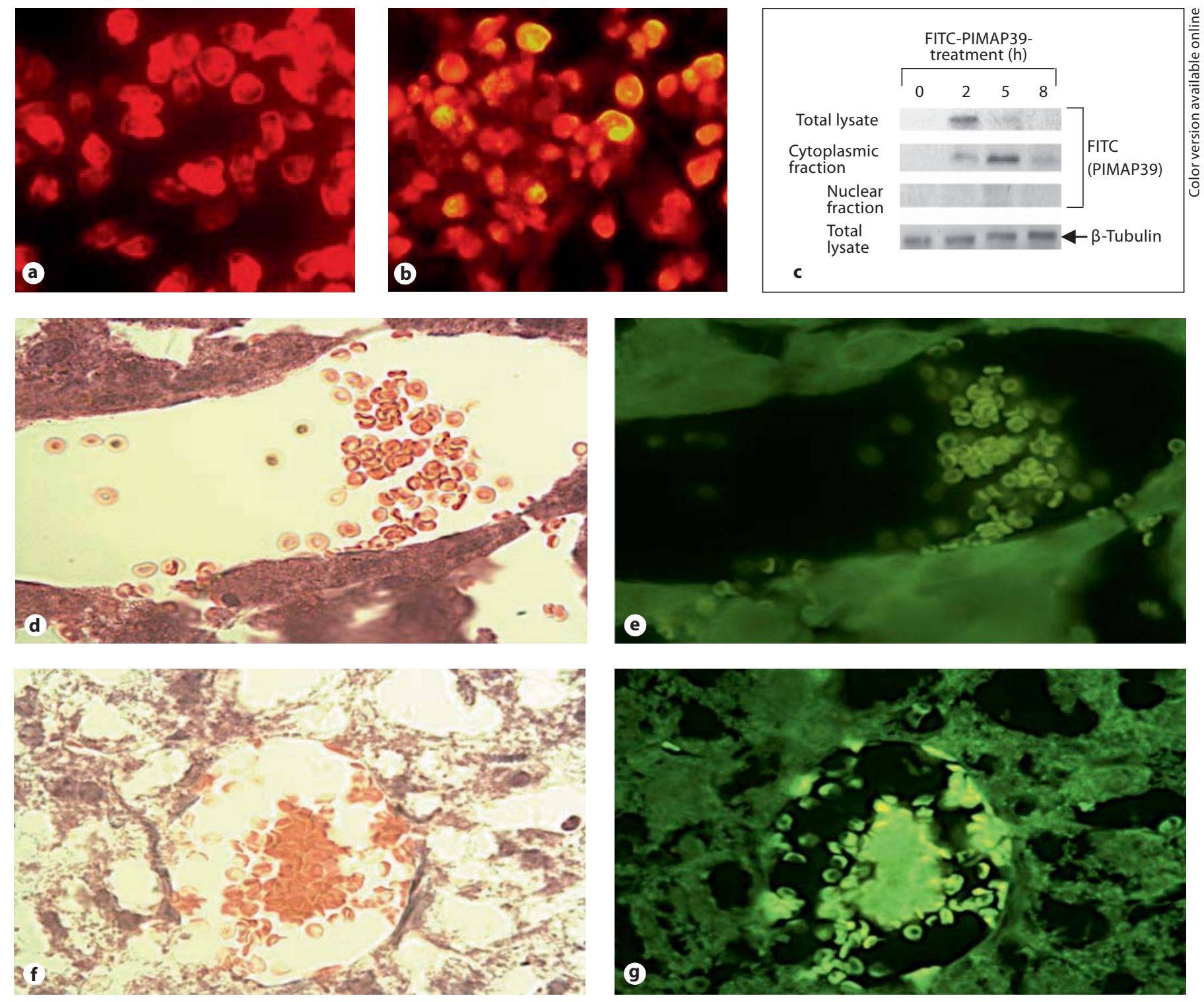

Fig. 4. FITC-labeled PIMAP39 in mouse primary macrophages (a-c) and mouse liver (tissue sections; $\mathbf{d}-\mathbf{g}$ ). Primary macrophage samples: cells $\left(5 \times 10^{3}\right)$ from 3 -month-old mice were seeded over cover slips $(22 \mathrm{~mm})$ in 6 -well plates, and were treated with 500 ng/ml FITC-PIMAP39 (b) or DMSO alone (a), and continuously incubated overnight. Cover slips were removed and cells were stained with $50 \mathrm{nM}$ LysoTracker Red DND-99 (Invitrogen) for $1.5 \mathrm{~h}$ and then air-dried. For the detection of PIMAP39 cellular location, primary macrophages were treated with $500 \mathrm{ng} / \mathrm{ml}$ FITC-PIMAP39. Protein extracts were collected from whole cells, cytoplasm or nuclei after peptide treatment at each time point $(0$, 2,5 and $8 \mathrm{~h}$ ). Thirty micrograms of lysate proteins were detected by Western blot with antibodies against FITC (sc-65218; Santa Cruz Biotechnology) and a-tubulin (C-20; Santa Cruz Biotechnology) as control (c). Tissue section samples: mice were treated with $1 \mathrm{mg}$ of FITC-PIMAP39 (f, $\mathbf{g})$ or DMSO alone as control $(\mathbf{d}, \mathbf{e})$, by tail vein injection as described. One hour after injection, mice

were sacrificed and their livers were harvested. Harvested livers were set in uniform orientations in molds using Histoprep (Fisher). Ten micrometer-thick cross-sections were cut and transferred with a paintbrush to glass slides followed by hematoxylin and eosin staining. Cross-sections were made at $-24^{\circ} \mathrm{C}$ using $\mathrm{HM} 505 \mathrm{E}$ cryostat (Microm). Both the treated cells and sections above were exposed to visible light for structure identification $(\mathbf{a}, \mathbf{d}, \mathbf{f})$ and fluorescent light for signal location (b, e, g) by Olympus BX40 microscope at magnifications of $200 \times(\mathbf{a}, \mathbf{b})$ or $1,000 \times(\mathbf{d}-\mathbf{g})$ and photographed. The FITC-PIMAP39-induced fluorescent signal in some primary macrophages (b) or in the leukocytes within the veins of the liver $(\mathbf{g})$ was observed. The images were taken with a MicroFIRE camera under uniform exposure time (1 s for fluorescent light, $30 \mathrm{~ms}$ for visible light). The data analysis was processed by the software Image-Pro plus 5.0. Multiple tests were done with similar results. The results from one test are presented here. 

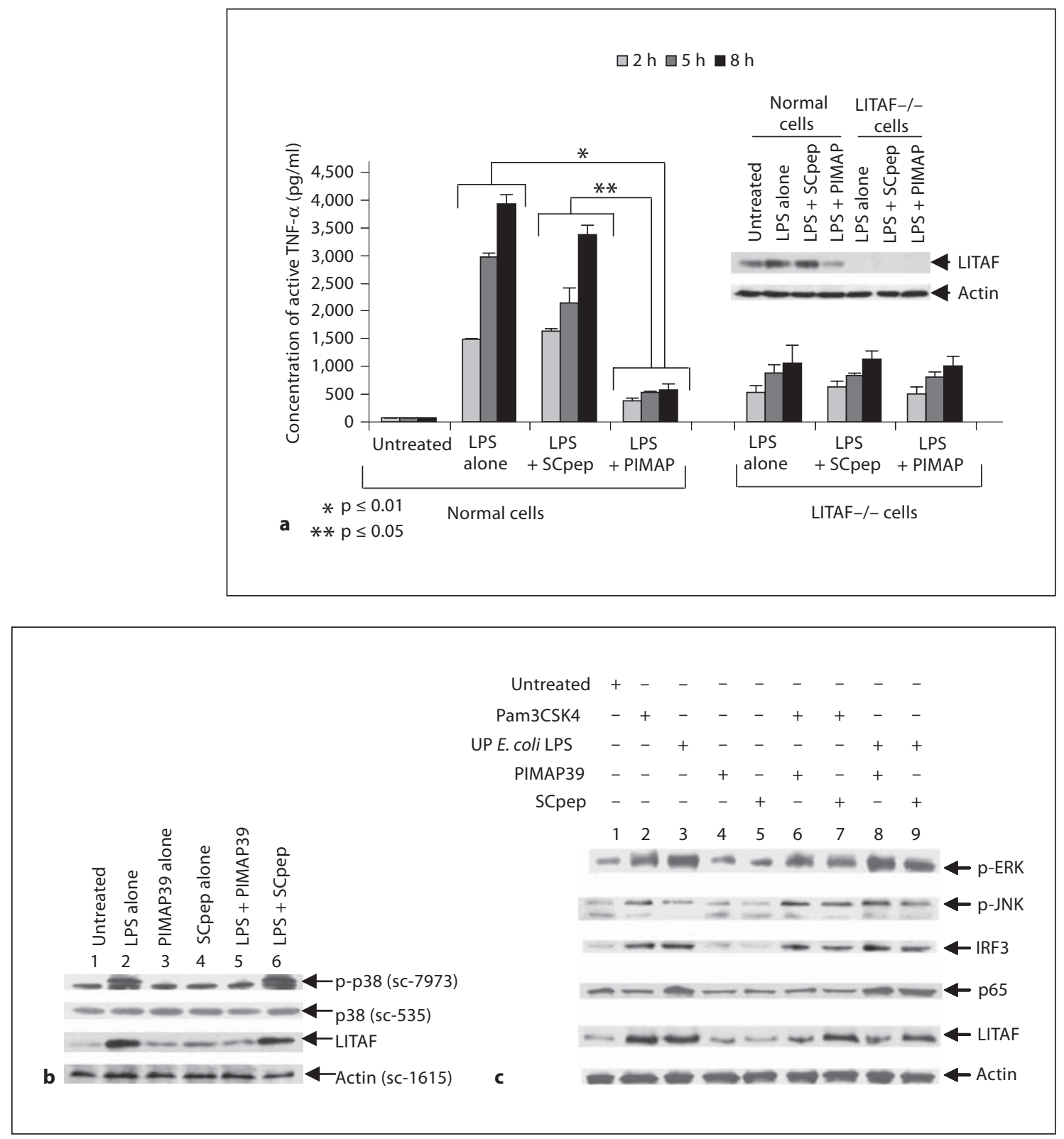

Fig. 5. Effects of PIMAP39 on LPS-induced TNF- $\alpha$ secretion. To assess PIMAP39 function, THP-1 cells and LITAF-/- primary macrophages were stimulated with $0.1 \mu \mathrm{g} / \mathrm{ml}$ E. coli LPS for $3 \mathrm{~h}$, washed with PBS and given fresh medium prior to treatment with $500 \mathrm{ng} / \mathrm{ml}$ FITC-PIMAP39 or SCpep as control. Two hundred microliters of supernatant was collected from each cell culture at each time point $(2,5$ and $8 \mathrm{~h}$ ) after treatment as described above. The supernatants were measured by ELISA (Abraxis) to see effects of PIMAP39 on TNF- $\alpha$ production. ELISA immunoreactivity was quantified using a microplate reader (Bio-Rad) and graphed (a). For genomic analysis of cells, the lysate from both THP-1 and LITAF-/- primary macrophages were detected by Western blot with antibodies against LITAF and actin. The result was attached (a). Additionally, lysates from each THP-1 sample were used for Western blot detection (b) with antibodies against p38 and p-p38, LITAF, and actin as control. Multiple tests were done with similar results. The results from 1 test are presented here. Furthermore, primary macrophages from wild-type mice (C57BL/6; The Jackson Lab) were treated with ultra pure E. coli LPS (UP E. coli LPS) or Pam3CSK4 plus PIMAP39 or SCpep as control over night. Cell lysate from each test sample was assayed by Western blot with antibodies against phosphoERK (sc-7976; Santa Cruz Biotechnology), phosphoJNK (No. 9251; Cell Signaling Technology), IRF3 (sc-9082; Santa Cruz Biotechnology), p65, LITAF or actin as control (c). 


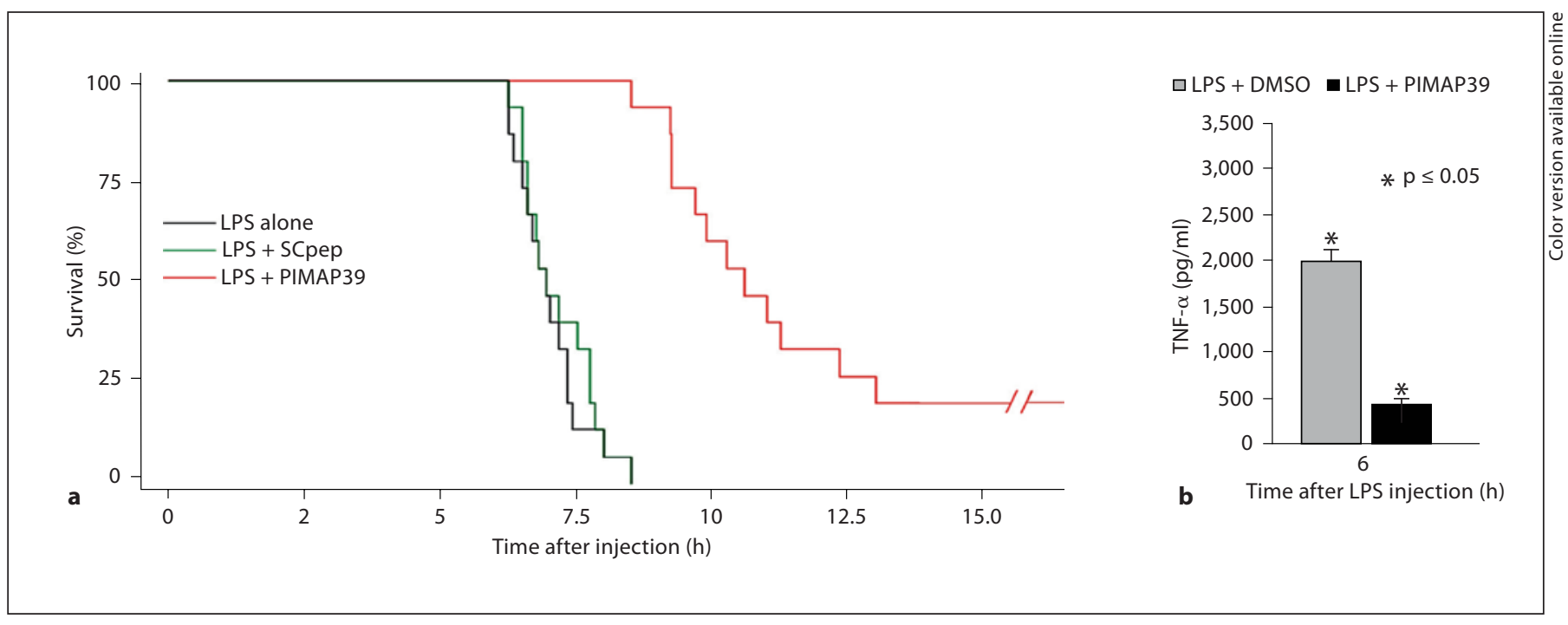

Fig. 6. Effect of PIMAP39 on LPS-induced endotoxic shock. a Weight-matched wild-type mice $(n=15)$ were injected intraperitoneally with a lethal dose of LPS (1 mg D-Gal + $12.5 \mathrm{ng}$ LPS per gram body weight) followed immediately by tail vein injection of DMSO, $40 \mu \mathrm{g} / \mathrm{g}$ SCpep as control or $40 \mu \mathrm{g} / \mathrm{g}$ PIMAP39. The treated mice were monitored for their behavior and mortality every hour. The survival time of each treated mouse was graphed. Significant differences were noted between the PIMAP39-treated mice and both the LPS alone and LPS + SCpep groups $(p<0.0001$, log-rank and Wilcoxon tests). b Blood was collected every $2 \mathrm{~h}$ after injection for a total duration of $6 \mathrm{~h}$. Mice were warmed under heating lamps to promote blood flow, and a small incision was made on the tail. About $10-50 \mathrm{nl}$ of blood was collected per animal at each time point $(2,4$ or $6 \mathrm{~h})$. Blood samples from mice within the same group were pooled. Red blood cells were removed from the sample via centrifugation at 5,000 rpm for $1 \mathrm{~min}$ using serum separator tubes (Fisher). Pooled plasma samples from the mice within each group were measured in triplets by ELISA (Abraxis) to see effects of PIMAP39 on TNF- $\alpha$ production. ELISA immunoreactivity was quantified using a microplate reader (Bio$\mathrm{Rad}$ ) and the results from the 6-hour mark were graphed (only the data for LPS + DMSO and LPS + PIMAP39 are presented here due to the similarity between LPS + DMSO and LPS + SCpep).

location of the LITAF-STAT6(B) complex into the nucleus significantly regulates transcription of several inflammatory cytokines including TNF- $\alpha[7,8]$. LPS-mediated p38 MAPK activation serves as an upstream kinase of LITAF which specifically activates LITAF gene expression [9].

p38 MAPK is activated by various growth factors and cytokines and its activity is suppressed by protein tyrosine phosphatases [11-13, 26]. In the human MTC cell line TT, SRIF stimulated the PTP activity which is associated with inhibition of proliferation and reduced MAPK activity. A dual-specificity phosphatase 1 was recently found to be essential for the control of $\mathrm{p} 38$ activation in LPS-activated macrophages and also to be important in regulating TNF as well as endotoxic shock after LPS treatment in mice [27]. Blockade of PTP activity with sodium orthovanadate has been shown to induce cell proliferation and increased p38 MAPK phosphorylation [28]. Our initial observation indicates that in addition to these effects, PTP activity inhibits LITAF promoter activity (fig. 1a). The current study 
has identified a possible link between PTP4A3 and LPSinduced p38 MAPK activation and its subsequent influence in LPS-induced cytokine production.

Although the role of PTP4A3 in cancer growth and metastasis is extensively analyzed [17], the putative function of PTP4A3 in LPS-induced inflammatory responses remains largely unknown. Our present data indicate that although PTP4A3 does not affect the activation of Akt, JAK, ERK or NF- $\kappa B$ p65, it selectively dephosphorylates LPS-induced p $38 \alpha$ MAPK phosphorylation in both THP1 and mouse primary macrophages. Recent studies show that PTPs are involved in the regulation of LPS-induced production of TNF- $\alpha[29,30]$, but the role of PTP in this system is poorly known.

In this study, a specific short peptide (PIMAP39) is found to mimic the activity of full-length PTP4A3 to dephosphorylate $\mathrm{p} 38 \alpha$ and to suppress LITAF/TNF- $\alpha$, production in response to LPS stimulation. The analysis of PIMAP39 shows that this unique sequence is located upstream of both the catalytic area and $\mathrm{SH} 2$ domain. Surprisingly, PIMAP39 peptide is able to easily penetrate into mouse primary macrophage cells in vitro and to be taken up by mouse white blood cells in vivo, without any cell delivery vehicle such as transfection agents. Indeed, treatment of mice with $40 \mu \mathrm{g} / \mathrm{g}$ of PIMAP39, or even a low concentration $(0.4 \mu \mathrm{g} / \mathrm{g})$, efficiently reduces septic shock symptoms associated with endotoxemia and prolongs survival time by about $4-5 \mathrm{~h}$ compared to controls, most likely due to the peptide efficiently downregulating LPS-induced TNF- $\alpha$ production. In support of its functional role in vivo, the analysis of blood samples from mice injected with PIMAP39 shows that these mice maintain a 78.3\% lower concentration of TNF- $\alpha$ compared to control mice that typically display a rapid increase in TNF- $\alpha$ production within $6 \mathrm{~h}$. The data suggest that mice treated with PIMAP39 significantly resist LPS-induced endotoxic shock most likely due to the suppression of TNF- $\alpha$ production.

Several studies indicated that mice lacking a gene such as poly (ADP-ribose) polymerase-1, or a receptor such as CC chemokine receptor 4 , are resistant to LPS-induced death, suggesting that these are involved in the regulation of the NF- $\kappa \mathrm{B}$ signaling pathway leading to synthesis of inflammatory mediators and the development of LPS-induced endotoxic shock [31, 32]. However, these studies provide only a limited explanation of the role of these factors in LPS-induced endotoxic shock. This study provides further building blocks to elucidate LPS-LITAF-TNF complex mechanism in demonstrating that (1) PTP4A3 affects LITAF and (2) LPS-induced death in mice is re- duced by $12.5 \%$ after PIMAP39 treatment, suggesting that PTP4A3 and its derived peptide, PIMAP39, can also affect LPS-induced endotoxemia. Furthermore, in wildtype peritoneal mouse macrophages, the addition of PIMAP39 did not change activation/phosphorylation of JNK, ERK, p65 or IRF3 in response to either TLR4 or TLR2 ligand, while PIMAP39 significantly affected LITAF activation suggesting that PIMAP39 prevents LPS-induced TNF- $\alpha$ production possibly by interfering with LITAF rather than NF- $\kappa$ B. Altogether, the present data indicate that PTP4A3 may be specifically regulating LITAF-dependent events in response to LPS stimulation, and that LITAF-independent genes would not be affected by PIMAP39 treatment of cells or by altering PTP4A3 function. Additionally, we identified the specific amino acid residue of PIMAP39 (100\% homologous) within a conserved region of multiple groups, including mouse, monkey and human, which will enable us to design additional animal models to further clarify the functional capabilities of PIMAP39 in alleviating the symptoms of LPS-induced diseases.

Current studies also demonstrate that LPS treatment of monocyte cells increases tyrosine phosphorylation of certain proteins, and that herbimycin A and genistein, general inhibitors of tyrosine kinases, markedly attenuated LPS-induced TNF- $\alpha$ expression both at the protein and mRNA levels. The ability of LPS to promote TNF- $\alpha$ production was further enhanced by treatment of cells with tyrosine phosphatase inhibitor, sodium orthovanadate. However, the timing of the LPS-dependent phosphorylation of proteins on tyrosine remains unclear. In the present paper we show that LPS stimulation induces an accumulation of PTP4A3 between $8-10 \mathrm{~h}$, after which its expression immediately decreases and eventually returns to its normal level. During this period, the maximal level of PTP4A3 coincides with no increase in the LPSinduced TNF- $\alpha$ protein rate, suggesting that LPS-induced PTP4A3 is the short-lived protein responsible for temporally inhibiting TNF- $\alpha$ production at this time point. Afterwards, TNF- $\alpha$ production resumes due to the rapid degradation or inactivation of PTP4A3. We also found that PTP4A3 is always expressed at a basal level, and while this is a low level for most cells, such as fibroblasts, its basal expression level in THP-1 or primary macrophage cells is relatively high [unpubl. data]. In addition, cotreatment of cells with LPS and orthovanadate augmented the LPS-induced expression of TNF- $\alpha$ (fig. $1 \mathrm{~b}-\mathrm{d}, 8-16 \mathrm{~h}$ ), suggesting that PTPs play an important role in the fine tuning of the immune response. Altogether, the data suggest that without PTP4A3, cells are 
Fig. 7. Diagram of a putative LPS-induced $\mathrm{PTP} / \mathrm{LITAF} / \mathrm{TNF}$ signaling pathway.

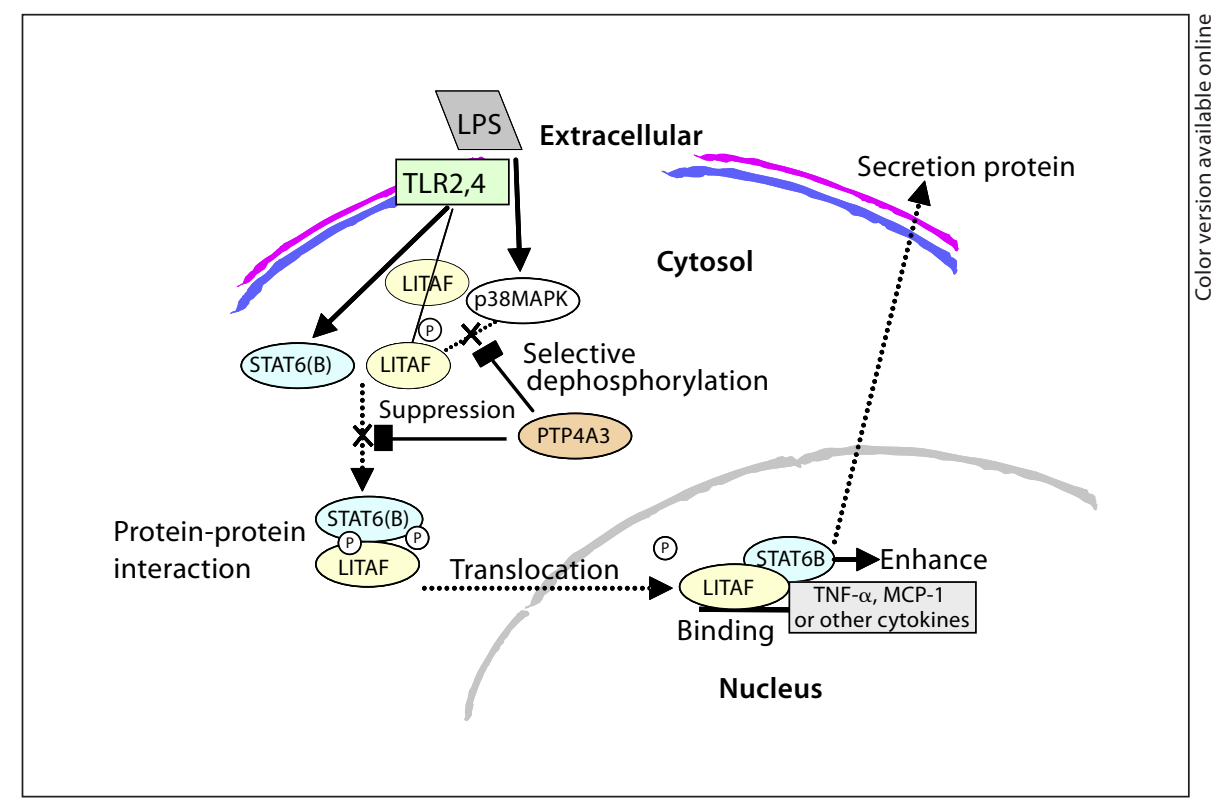

more sensitive to LPS stimulation, but further work is required to shed new light on the mechanisms involved.

A novel role for PTP4A3 in LPS-induced cytokine activation is proposed and its interactions are depicted in the signal pathway in figure 7. Both LITAF and STAT6B are nuclear transcription factors that regulate expression of cytokines such as TNF- $\alpha$, IL-1 $\alpha$, IL-1 $\beta$, MCP-1, MCP2 and VEGF. They are induced by Porphyromonas gingivalis LPS via TLR-2 or by E. coli LPS via TLR-4. Their production is initially sequestered in the cytoplasm. Our hypothesis is that they are phosphorylated by $\mathrm{p} 38 \mathrm{MAP}$ kinase prior to protein-protein interactions aimed at forming a complex. This would lead to translocation of the molecules to the nucleus. In the nucleus, the complex would bind to the LITAF-specific sequence (CTCCC) present on various cytokine promoter genes and thus activates their transcription. However, overexpression of PTP4A3 would selectively dephosphorylate p38 $\alpha$, thereby indirectly suppressing LPS-induced LITAF/TNF- $\alpha$ production in the cytoplasm. Ultimately, a better understanding of this pathway will be instrumental for the design and development of target agents aimed at pharmacological interventions in inflammatory processes.

\section{Acknowledgment}

This work was supported by grants from the National Institute of Dental and Craniofacial Research DE RO1 DE14079 (S.A.).

\section{References}

Young LR, Borchers MT, Allen HL, Gibbons RS, McCormack FX: Lung-restricted macrophage activation in the pearl mouse model of Hermansky-Pudlak syndrome. J Immunol 2006;176:4361-4368.

-2 Rushworth SA, Taylor A, Langa S, MacEwan DJ: TNF signaling gets FLIPped off: TNFinduced regulation of FLIP. Cell Cycle 2008; 7:194-199.

3 Zanin-Zhorov A, Tal-Lapidot G, Cahalon L, Cohen-Sfady M, Pevsner-Fischer M, Lider O, Cohen IR : Cutting edge: T cells respond to lipopolysaccharide innately via TLR4 signaling. J Immunol 2007;179:41-44.
4 Khadaroo RG, Kapus A, Powers KA, Cybulsky MI, Marshall JC, Rotstein OD: Oxidative stress reprograms lipopolysaccharide signaling via Src kinase-dependent pathway in RAW 264.7 macrophage cell line. J Biol Chem 2003;278:47834-47841

5 Lin WN, Luo SF, Lee CW, Wang CC, Wang JS, Yang CM: Involvement of MAPKs and NF-kappaB in LPS-induced VCAM-1 expression in human tracheal smooth muscle cells. Cell Signal 2007;19:1258-1267. 
6 Handley ME, Thakker M, Pollara G, Chain BM, Katz DR: JNK activation limits dendritic cell maturation in response to reactive oxygen species by the induction of apoptosis. Free Radic Biol Med 2005;38:1637-1652.

$>7$ Tang X, Fenton MJ, Amar S: Identification and functional characterization of a novel binding site on TNF- $\alpha$ promoter. Proc Natl Acad Sci USA 2003;100:4096-4101.

$>8$ Tang X, Marciano DL, Leeman SE, Amar S: LPS induces the interaction of a transcription factor, LPS-induced TNF- $\alpha$ factor, and STAT6(B) with effects on multiple cytokines. Proc Natl Acad Sci USA 2005;102:51325137.

-9 Tang X, Metzger D, Leeman S, Amar S: LPSinduced TNF- $\alpha$ factor (LITAF)-deficient mice express reduced LPS-induced cytokine: evidence for LITAF-dependent LPS signaling pathways. Proc Natl Acad Sci USA 2006; 103:13777-13782.

10 Bamford S, Ryley H, Jackson SK: Highly purified lipopolysaccharides from Burkholderia cepacia complex clinical isolates induce inflammatory cytokine responses via TLR4mediated MAPK signalling pathways and activation of NF-кB. Cell Microbiol 2007;9: 532-543.

-11 Baeza-Raja B, Munoz-Canoves P:p38MAPKinduced nuclear factor-kappaB activity is required for skeletal muscle differentiation: role of interleukin-6. Mol Biol Cell 2004;15: 2013-2026.

- 12 Mizuno T, Hisamoto N, Terada T, Kondo T, Adachi M, Nishida E, Kim DH, Ausubel FM, Matsumoto K: The Caenorhabditis elegans MAPK phosphatase VHP-1 mediates a novel JNK-like signaling pathway in stress response. EMBO J 2004;23:2226-2234.

-13 Tanoue T, Adachi M, Moriguchi T, Nishida E: A conserved docking motif in MAP kinases common to substrates, activators and regulators. Nat Cell Biol 2000;2:110-116.

14 Chi H, Barry SP, Roth RJ, Wu JJ, Jones EA, Bennett AM, Flavell RA: Dynamic regulation of pro- and anti-inflammatory cytokines by MAPK phosphatase 1 (MKP-1) in innate immune responses. Proc Natl Acad Sci USA 2006; 103:2274-2279.

-15 Fiordalisi JJ, Keller PJ, Cox AD: PRL tyrosine phosphatases regulate rho family GTPases to promote invasion and motility. Cancer Res 2006;66:3153-3161.
-16 Zeng Q, Si X, Horstmann H, Xu Y, Hong W, Pallen CJ: Prenylation-dependent association of protein-tyrosine phosphatases PRL$1,-2$, and -3 with the plasma membrane and the early endosome. J Biol Chem 2000;275: 21444-21452.

17 Saha S, Bardelli A, Buckhaults P, Velculescu VE, Rago C, St Croix B, Romans KE, Choti MA, Lengauer C, Kinzler KW, Vogelstein B: A phosphatase associated with metastasis of colorectal cancer. Science 2001;294:13431346.

18 Shimomura H, Matsuura M, Saito S, Hirai Y, Isshiki Y, Kawahara K: Lipopolysaccharide of Burkholderia cepacia and its unique character to stimulate murine macrophages with relative lack of interleukin-1 $\beta$-inducing ability. Infect Immun 2001;69:3663-3669.

19 Greenwel P, Hu W, Kohanski RA, Ramirez F: Tyrosine dephosphorylation of nuclear proteins mimics transforming growth factor $\beta 1$ stimulation of $\alpha 2(\mathrm{I})$ collagen gene expression. Mol Cell Biol 1995;15:6813-6819.

20 Saudemont A, Hamrouni A, Marchetti P, Liu J, Jouy N, Hetuin D, Colucci F, Quesnel B: Dormant tumor cells develop cross-resistance to apoptosis induced by CTLs or imatinib mesylate via methylation of suppressor of cytokine signaling 1. Cancer Res 2007;67: 4491-4498.

21 Yang L, Amann JM, Kikuchi T, Porta R, Guix M, Gonzalez A, Park KH, Billheimer D, Arteaga CL, Tai HH, DuBois R, Carbone DP, Johnson DH: Inhibition of epidermal growth factor receptor signaling elevates 15-hydroxyprostaglandin dehydrogenase in nonsmall-cell lung cancer. Cancer Res 2007;67: 5587-5593.

-22 Hsieh YH, Wu TT, Huang CY, Hsieh YS, Hwang JM, Liu JY: p38 mitogen-activated protein kinase pathway is involved in protein kinase $\mathrm{C} \alpha$-regulated invasion in human hepatocellular carcinoma cells. Cancer Res 2007;67:4320-4327

23 Tang X, Molina M, Amar S: p53 short peptide (p53pep164) regulates lipopolysaccharideinduced tumor necrosis factor-alpha factor/ cytokine expression. Cancer Res 2007;67: 1308-1316.

24 Sossey-Alaoui K, Safina A, Li X, Vaughan MM, Hicks DG, Bakin AV, Cowell JK: Downregulation of WAVE3, a metastasis promoter gene, inhibits invasion and metastasis of breast cancer cells. Am J Pathol 2007;170: 2112-2121.
25 Munoz JJ, Tarrega C, Blanco-Aparicio C, Pulido R: Differential interaction of the tyrosine phosphatases PTP-SL, STEP and HePTP with the mitogen-activated protein kinases ERK $1 / 2$ and $\mathrm{p} 38 \alpha$ is determined by a kinase specificity sequence and influenced by reducing agents. Biochem J 2003;372:193201.

26 Chiarugi P, Cirri P, Taddei ML, Giannoni E, Fiaschi T, Buricchi F, Camici G, Raugei G, Ramponi G: Insight into the role of low molecular weight phosphotyrosine phosphatase (LMW-PTP) on platelet-derived growth factor receptor (PDGF-r) signaling. LMW-PTP controls PDGF-r kinase activity through TYR-857 dephosphorylation. J Biol Chem 2002;277:37331-37338.

$\checkmark 27$ Hammer M, Mages J, Dietrich H, Servatius A, Howells N, Cato ACB, Lang R: Dual specificity phosphatase 1 (DUSP1) regulates a subset of LPS-induced genes and protects mice from lethal endotoxin shock. J. Exp Med 2006;203:15-20.

28 Zatelli MC, Piccin D, Tagliati F, Bottoni A, Luchin A, degli Uberti EC: SRC homology2-containing protein tyrosine phosphatase1 restrains cell proliferation in human medullary thyroid carcinoma. Endocrinology 2005;146:2692-2698.

29 Kim HY, Park EJ, Joe EH, Jou I: Curcumin suppresses Janus kinase-STAT inflammatory signaling through activation of Src homology 2 domain-containing tyrosine phosphatase 2 in brain microglia. J Immunol 2003;171: 6072-6079.

30 Mendoza H, Campbell DG, Burness K, Hastie J, Ronkina N, Shim JH, Arthur JS, Davis RJ, Gaestel M, Johnson GL, Ghosh S, Cohen $\mathrm{P}$ : Roles for TAB1 in regulating the IL-1-dependent phosphorylation of the TAB3 regulatory subunit and activity of the TAK $1 \mathrm{com}$ plex. Biochem J 2008;409:711-722.

-31 Oliver FJ, Menissier-de Murcia J, Nacci C, Decker P, Andriantsitohaina R, Muller S, de la Rubia G, Stoclet JC, de Murcia G: Resistance to endotoxic shock as a consequence of defective NF- $\kappa \mathrm{B}$ activation in poly (ADP-ribose) polymerase-1 deficient mice. EMBO J 1999;18:4446-4454.

>32 Chvatchko Y, Hoogewerf AJ, Meyer A, Alouani S, Juillard P, Buser R, Conquet F, Proudfoot AE, Wells TN, Power CA: A key role for CC chemokine receptor 4 in lipopolysaccharide-induced endotoxic shock. J Exp Med 2000;191:1755-1764. 